\title{
Hyperuricemia is a biomarker of early mortality in patients with chronic obstructive pulmonary disease
}

This article was published in the following Dove Press journal:

International Journal of COPD

26 November 2015

Number of times this article has been viewed

\section{Xin Zhang \\ Lijie Liu \\ Rui Liang \\ Shoude Jin}

Department of Respiratory Medicine, Fourth Affiliated Hospital of Harbin Medical University, Harbin, People's Republic of China
Correspondence: Shoude Jin Department of Respiratory Medicine, Fourth Affiliated Hospital of Harbin Medical University, 37 Yiyuan Street, Harbin I5000I, People's Republic of China

Email jsdemail@yeah.net
Abstract: Patients with chronic obstructive pulmonary disease (COPD) are often at high risk of early death. Identification of prognostic biomarkers for COPD may aid in improving their survival by providing early strengthened therapy for high-risk patients. In the present study, we investigated the prognostic role of hyperuricemia at baseline on the prognosis of patients with COPD. Thirty-four patients with COPD with hyperuricemia were matched $(1: 2)$ to 68 patients with COPD without hyperuricemia and of similar age and sex. Data from those patients with COPD were evaluated retrospectively. The role of hyperuricemia on mortality was first analyzed using the Kaplan-Meier method, and multivariate Cox regression model was then used to evaluate the prognostic significance of hyperuricemia in patients with COPD. Hyperuricemia was not associated with other baseline characteristics in patients with COPD. Kaplan-Meier survival curve showed that patients with COPD with hyperuricemia had higher risk of mortality compared with patients with normouricemia, and the $P$-value for log-rank test was 0.005 . In univariate analysis, hyperuricemia was associated with higher risk of mortality in patients with COPD (hazard ratio $=2.29,95 \% \mathrm{CI}=1.07-4.88, P=0.032$ ). In the multivariate analysis, hyperuricemia was independently associated with higher risk of mortality in patients with COPD (hazard ratio $=2.68,95 \% \mathrm{CI}=1.18-6.09, P=0.019$ ). In conclusion, hyperuricemia is a promising biomarker of early mortality in patients with COPD.

Keywords: hyperuricemia, chronic obstructive pulmonary disease, mortality, uric acid

\section{Introduction}

Chronic obstructive pulmonary disease (COPD) is a major contributor to morbidity worldwide, and it has affected millions of people., ${ }^{1,2}$ COPD is still a smoking-related disorder and one of the most common causes of death. ${ }^{3}$ Although COPD mainly affected persons in the age group of 40-50 years, the pathogenesis of this disease can begin in early life and result in early death. ${ }^{4,5}$ There are several risk factors associated with COPD in later life, such as smoking, diabetes, and vitamin D deficiency. ${ }^{6-8}$ Since COPD is associated with increased early mortality compared with general population, development of new treatments aiming to prolong their lives is important. In addition, identification of clinical characteristics predicting mortality is also helpful to improve the efficacy of current treatments for an individual patient. ${ }^{9,10}$ Identification of prognostic biomarker for COPD may aid in improving the survival by providing early strengthened therapy for high-risk patients. So far, several prognostic factors for mortality have been identified for patients with COPD, such as acute exacerbations, cerebrovascular disease, diabetes, and alcohol abuse. ${ }^{11-14}$ Uric acid (UA) is an end product of the metabolic breakdown of purine nucleotides. ${ }^{15,16}$ Previous studies 
have shown that UA has a particular importance in human body for its antioxidant nature. ${ }^{15,16}$ However, UA also has the contrary effect and is a proinflammatory factor. ${ }^{17,18}$ The proinflammatory effect of UA is more profound in those with high serum UA levels. ${ }^{17,18}$ Hyperuricemia is defined as serum UA levels $>420 \mu \mathrm{mol} / \mathrm{L}$ in men or $>360 \mu \mathrm{mol} / \mathrm{L}$ in women. ${ }^{17}$ Compared with individuals with normouricemia, individuals with hyperuricemia have more inflammation and oxidative stress injuries. Previous studies have suggested that hyperuricemia is strongly associated with increased risks of cardiovascular diseases and all-cause mortality. ${ }^{19,20}$ Although several studies have been performed to assess the association between UA and COPD, the cause-effect relationship between hyperuricemia and COPD is still unknown. ${ }^{21-23}$ Currently, the prognostic role of hyperuricemia at baseline on the prognosis of patients with COPD is still unclear. In present study, we analyzed data from a retrospective, observational study that investigated the prognostic role of hyperuricemia at baseline on the mortality in patients with COPD.

\section{Materials and methods Study design}

The purpose of this study was to assess the prognostic role of hyperuricemia at baseline on the mortality in patients with COPD. The medical records of patients with diagnosed COPD who were treated at our hospital and admitted into our hospital for therapy were searched retrospectively from July 30 , 2011 to June 30, 2012. For the patients to be included into our study, the diagnosis of COPD in those patients must be based on the World Health Organization Global Initiative for Chronic Obstructive Lung Disease (GOLD) criteria. ${ }^{24}$ All were diagnosed on the basis of clinical history, physical examination, chest computed tomography, and pulmonary function tests in accordance with the GOLD criteria for the diagnosis and severity of COPD. ${ }^{25}$ In addition, the data of UA and data of follow-up must be available from those patients. Patients with a history of chronic kidney disease, receiving diuretic treatment, or with lowering UA therapy that could be associated with the abnormal biomarker profile were all excluded. Patients with chronic lung conditions such as asthma, bronchiectasis, and interstitial lung disease were also excluded. Thirty-four patients with COPD with hyperuricemia were matched (1:2) to 68 patients with COPD without hyperuricemia and of similar age and sex. Therefore, 102 patients with COPD were finally included into our study.

The study was approved by the local ethical committee of our hospital. All participants provided informed consent. Patients without available data of serum UA levels or outcomes of follow-up were also excluded from the study. The data of clinical characteristics were retrieved from the medical records at our hospital or by contacting the patients. These data used in our study mainly included date of diagnosis, area of resident, age, sex, smoking, times of acute exacerbations, comorbidities, stages of GOLD, outcomes of follow-up, and serum UA levels. The UA levels were obtained within 1-2 days after admission. The UA levels were determined with uricase-peroxidase method using an autoanalyzer (Hitachi 747; Hitachi Ltd., Tokyo, Japan) at the central laboratory of our hospital. Hyperuricemia was defined as serum UA levels $>420 \mu \mathrm{mol} / \mathrm{L}$ in men and women. On the contrary, normouricemia was defined as serum UA levels $<420 \mu \mathrm{mol} / \mathrm{L}$ in men and women.

\section{Outcomes and follow-up}

The primary end outcome was overall survival, defined as from the time of definite diagnosis to death of any cause or the final time of follow-up (in months). The second outcome was 1-year mortality after discharge from hospital. Follow-up evaluations were performed through the medical records or by contacting the patients and their relatives. The follow-up of patients with COPD was performed every 3 months. The mortality was first identified from those medical records, and then investigators ascertained it by direct confirmation by contacting a family member. However, the cause of death was not registered since the cause of death was not available from part of those patients.

\section{Statistical analysis}

Continuous variables were expressed as mean \pm standard deviation or as median and interquartile range (25\%-75\%). The associations between hyperuricemia and clinical parameters were assessed using chi-square test. The impact of hyperuricemia on survival of patients with COPD was evaluated using the Kaplan-Meier method and the log-rank test. For the primary analysis, the hazard ratio (HR) with 95\% confidence interval $(95 \% \mathrm{CI})$ was reported as relative risk of mortality and was calculated through Cox regression models of univariate analysis and multivariate analysis. The covariates used in the present multivariate analysis were age, sex, smoking, comorbidities, and GOLD stages. All statistical analyses were performed using the STATA version 12.0. A two-sided $P>0.05$ was considered statistically significant.

\section{Results}

\section{Characteristics of patients with COPD}

Overall, 57 male and 45 female patients with COPD were included in the retrospective cohort study. The mean age at 
diagnosis of those patients with COPD was $47.5 \pm 10.5$ years. Comparison of clinical characteristics according to serum UA levels are shown in Table 1. None of these clinical characteristics were associated with hyperuricemia (Table 1).

\section{Hyperuricemia and mortality}

Kaplan-Meier survival curve showed that patients with COPD with hyperuricemia had higher risk of mortality compared with patients with normouricemia, and the $P$-value for log-rank test was 0.005 (Figure 1). In univariate analysis, hyperuricemia was associated with higher risk of mortality in patients with COPD ( $\mathrm{HR}=2.29,95 \% \mathrm{CI}=1.07-4.88$, $P=0.032$ ) (Table 2). Apart from hyperuricemia, advanced GOLD stage was also associated with higher risk of mortality in patients with COPD $(\mathrm{HR}=3.02,95 \% \mathrm{CI}=1.32-6.91$, $P=0.009$ ). However, other factors were not associated with the risk of mortality in patients with COPD (Table 2).

In the multivariate analysis, hyperuricemia was independently associated with higher risk of mortality in patients with COPD (HR $=2.68,95 \%$ CI $=1.18-6.09, P=0.019)$ (Table 2).

The 1-year mortality rate after discharge from hospital in the hyperuricemia group was $14.7 \%$, which was higher than that in the normouricemia group (5.9\%), but there was no statistically obvious difference $(P=0.15)$.

\section{Discussion}

COPD is associated with increased early mortality compared with the general population, and identification of

Table I Clinical characteristics of patients in the retrospective study

\begin{tabular}{|c|c|c|c|}
\hline Factors & Hyperuricemia & Normouricemia & $P$-value \\
\hline Subjects (n) & 34 & 68 & \\
\hline \multicolumn{4}{|l|}{ Age, n (\%) } \\
\hline$<65$ years & 15 (44.1\%) & 31 (45.6\%) & \multirow[t]{2}{*}{0.88} \\
\hline$\geq 65$ years & 19 (55.9\%) & 37 (54.4\%) & \\
\hline \multicolumn{4}{|l|}{ Sex, n (\%) } \\
\hline Female & 15 (44.1\%) & 30 (44.1\%) & \multirow[t]{2}{*}{0.88} \\
\hline Male & $19(55.9 \%)$ & 38 (55.9\%) & \\
\hline \multicolumn{4}{|c|}{ Current smokers, n (\%) } \\
\hline No & $22(64.7 \%)$ & $4 \mathrm{l}(60.3 \%)$ & \multirow[t]{2}{*}{0.65} \\
\hline Yes & $12(35.3 \%)$ & $27(39.7 \%)$ & \\
\hline \multicolumn{4}{|c|}{ Diabetes, n (\%) } \\
\hline No & $5(14.7 \%)$ & 18 (26.5\%) & \multirow[t]{2}{*}{0.18} \\
\hline Yes & $29(85.3 \%)$ & $50(73.5 \%)$ & \\
\hline \multicolumn{4}{|c|}{ GOLD stages, n (\%) } \\
\hline $\mathrm{I} / \mathrm{II}$ & $17(50.0 \%)$ & 37 (54.4\%) & \multirow[t]{2}{*}{0.67} \\
\hline III/IV & $17(50.0 \%)$ & $31(45.6 \%)$ & \\
\hline Death within & $5(14.7 \%)$ & $4(5.9 \%)$ & 0.15 \\
\hline I year, n (\%) & & & \\
\hline
\end{tabular}

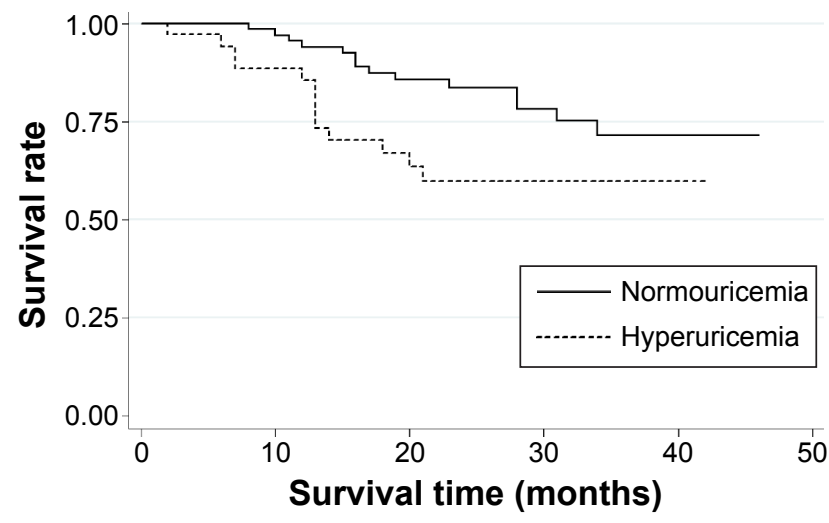

Figure I Kaplan-Meier survival curve assessing the influence of hyperuricemia on mortality in patients with COPD.

clinical characteristics predicting mortality is also helpful to improve the efficacy of current treatments for individual patient. ${ }^{5,9,12,26}$ For patients with risk factors of early mortality, such as vitamin D deficiency, asthma, and diabetes, early strengthened therapy may be recommended to improve their survival. ${ }^{2,8,27-30}$

In the present study, we investigated the prognostic role of hyperuricemia at baseline on the prognosis of patients with COPD. Thirty-four patients with COPD with hyperuricemia were matched (1:2) to 68 patients with COPD without hyperuricemia and of similar age and sex. Kaplan-Meier survival curve showed that patients with COPD with hyperuricemia had higher risk of mortality compared with patients with normouricemia, and the $P$-value for log-rank test was 0.005 (Figure 1). In addition, hyperuricemia was associated with higher risk of mortality in patients with COPD in both univariate analysis and multivariate analysis. Therefore, our study suggests that hyperuricemia may be a promising biomarker of early mortality in patients with COPD. However, given that the cause-effect relationship between UA and COPD is not established, more studies are needed to explore the possible cause-effect relationship between

Table 2 Univariate and multivariate Cox regression analysis evaluating the effect of hyperuricemia and confounders on mortality

\begin{tabular}{|c|c|c|c|c|}
\hline & \multicolumn{2}{|c|}{ Univariate analysis } & \multicolumn{2}{|c|}{ Multivariate analysis } \\
\hline & HR (95\% Cl) & $P$-value & HR (95\% Cl) & $P$-value \\
\hline Age ( $\geq 65$ years) & $1.13(0.52-2.44)$ & 0.32 & & \\
\hline Sex (female) & $\mathrm{I} .33(0.6 \mathrm{I}-2.90)$ & 0.48 & & \\
\hline Smoking & I.I5 (0.53-2.48) & 0.72 & & \\
\hline Diabetes & $2.00(0.90-4.45)$ & 0.089 & $1.75(0.66-4.67)$ & 0.262 \\
\hline GOLD stage (III/IV) & $3.02(\mid .32-6.91)$ & 0.009 & $2.41(0.95-6.15)$ & 0.065 \\
\hline Hyperuricemia & $2.29(1.07-4.88)$ & 0.032 & $2.68(1.18-6.09)$ & 0.019 \\
\hline
\end{tabular}

Abbreviations: HR, hazard ratio; GOLD, Global Initiative for Chronic Obstructive Lung Disease; $\mathrm{Cl}$, confidence interval. 
UA and COPD and the prognostic role of hyperuricemia in patients with COPD.

Previous studies have shown that UA has a particular importance in human body for its antioxidant nature. ${ }^{15,16}$ However, UA also has the contrary effect and is a proinflammatory factor, ${ }^{17,18}$ especially in those with high serum UA levels. ${ }^{17,18}$ COPD is associated with inflammation and oxidative stress, both of which have important roles in the pathogenesis of COPD.${ }^{31-33}$ Compared with individuals with normouricemia, individuals with hyperuricemia have more inflammation and oxidative stress injuries, which can further increase the severity and progression of COPD. ${ }^{17,34}$ Therefore, hyperuricemia may be involved in the aggravation of COPD by increasing inflammation and oxidative stress in the body, which is a plausible explanation for the association between hyperuricemia and early mortality in patients with COPD. In addition, hyperuricemia is a risk factor for cardiovascular disease including heart failure. ${ }^{19,20}$ Since cardiovascular diseases are common comorbidities of COPD, hyperuricemia may also result in higher risk of early mortality by promoting the progression of cardiovascular diseases existed in patients with COPD.

There were also several studies investigating the roles of UA in patients with COPD..$^{21,23,35-37}$ The study by Sato et al assessed the prognostic role of the ratio between the serum concentration of UA and creatinine in 91 patients with COPD, which found that the ratio appeared to be a reliable marker of prognosis. ${ }^{36}$ The study by Garcia-Pachon et al found that the ratio between the serum concentration of UA and creatinine was associated with lung function in patients with COPD. ${ }^{23}$ Other studies found that there were increased levels of UA in patients with COPD ${ }^{31,37}$ However, none of these studies assessed the association between hyperuricemia and early mortality in patients with COPD. In 2014, a study by Bartziokas et al showed that high UA levels were an independent predictor of 30-day mortality in patients with COPD, but not of 1-year mortality. ${ }^{35}$ The outcomes of our study showed that hyperuricemia is a biomarker of higher risk of mortality in patients with COPD, which provides interesting finding and valuable information for future studies. From the data mentioned earlier, it can be concluded that the cost of assessment of serum UA level is low, but it is a very useful biomarker in identifying high-risk patients with COPD, who may benefit from intensive management.

Though our study had several new findings, several limitations must be considered when interpreting the results from the study. First, our study was a retrospective cohort study. Though we matched patients with hyperuricemia to those without hyperuricemia and of similar age and sex, there were still other possible risks of bias. One of them was the bias caused by residual confounding factors. The retrospective design in our study did not allow us to consider all possible confounding factors in the multivariate analysis. Thus, to get a more precise evaluation on the prognostic role of hyperuricemia in patients with COPD, further studies with prospective are needed. In our study, we excluded patients with chronic kidney disease. However, the serum UA can be influenced by the glomerular filtration rate, which is also associated with mortality risk. Owing to the retrospective design, we did not add the glomerular filtration rate as a covariate, which was a limitation of our study. Second, there were only 34 patients with COPD with hyperuricemia and 68 matched patients with COPD without hyperuricemia in the study. The limited number of recruited participants was an obvious limitation, which may impair the quality of evidence in our study. More studies with large number of participants are needed to confirm the findings from this study. Hyperuricemia has been identified as a risk factor of cardiovascular disease in previous literature. Owing to the limited number of participants in our study, we were unable to assess whether hyperuricemia could be a risk factor for cardiovascular disease in patients with COPD. Future studies with large number of participants are needed to explore it. Finally, owing to the lack of relative data and small number of included participants, our study did not analyze the possible different prognostic roles of hyperuricemia in patients with COPD receiving different treatment regimens. The prognostic role of hyperuricemia in patients with COPD may be different in patients receiving different treatment regimens, which is very important for the clinical application of hyperuricemia as a useful prognostic biomarker. Future studies are needed to further analyze the possible different prognostic roles of hyperuricemia in patients with COPD receiving different treatment regimens.

\section{Conclusion}

In conclusion, hyperuricemia is a promising biomarker of early mortality in patients with COPD. However, more prospective cohort studies with large number of participants are needed to further identify the prognostic role of hyperuricemia in patients with COPD.

\section{Disclosure}

The authors report no conflicts of interest in this work.

\section{References}

1. Postma DS, Bush A, van den Berge M. Risk factors and early origins of chronic obstructive pulmonary disease. Lancet. 2015;385(9971): 899-909. 
2. Smith MC, Wrobel JP. Epidemiology and clinical impact of major comorbidities in patients with COPD. Int J Chron Obstruct Pulmon Dis. 2014;9:871-888.

3. Liu Y, Croft JB, Anderson LA, Wheaton AG, Presley-Cantrell LR, FordES. The association of chronic obstructive pulmonary disease, disability, engagement in social activities, and mortality among US adults aged 70 years or older, 1994-2006. Int J Chron Obstruct Pulmon Dis. 2014;9:75-83.

4. Drummond MB, Hansel NN, Connett JE, Scanlon PD, Tashkin DP, Wise RA. Spirometric predictors of lung function decline and mortality in early chronic obstructive pulmonary disease. Am J Respir Crit Care Med. 2012;185(12):1301-1306.

5. Terzano C, Conti V, Di Stefano F, et al. Comorbidity, hospitalization, and mortality in COPD: results from a longitudinal study. Lung. 2010;188(4): 321-329.

6. Zhang LL, Gong J, Liu CT. Vitamin D with asthma and COPD: not a false hope? A systematic review and meta-analysis. Genet Mol Res. 2014;13(3):7607-7616.

7. Autier P, Boniol M, Pizot C, Mullie P. Vitamin D status and ill health: a systematic review. Lancet Diabetes Endocrinol. 2014;2(1):76-89.

8. Afzal S, Lange P, Bojesen SE, Freiberg JJ, Nordestgaard BG. Plasma 25-hydroxyvitamin $\mathrm{D}$, lung function and risk of chronic obstructive pulmonary disease. Thorax. 2014;69(1):24-31.

9. Puhan MA, Siebeling L, Zoller M, Muggensturm P, ter Riet G. Simple functional performance tests and mortality in COPD. Eur Respir J. 2013; 42(4):956-963.

10. Moberg M, Vestbo J, Martinez G, et al. Validation of the i-BODE index as a predictor of hospitalization and mortality in patients with COPD participating in pulmonary rehabilitation. COPD. 2014;11(4) 381-387.

11. Perera PN, Armstrong EP, Sherrill DL, Skrepnek GH. Acute exacerbations of COPD in the United States: inpatient burden and predictors of costs and mortality. COPD. 2012;9(2):131-141.

12. Ryynanen OP, Soini EJ, Lindqvist A, Kilpelainen M, Laitinen T. Bayesian predictors of very poor health related quality of life and mortality in patients with COPD. BMC Med Inform Decis Mak. 2013;13:34.

13. Papaioannou AI, Bartziokas K, Loukides S, et al. Cardiovascular comorbidities in hospitalised COPD patients: a determinant of future risk? Eur Respir J. Epub 2015 Apr 16.

14. Riza AL, Pearson F, Ugarte-Gil C, et al. Clinical management of concurrent diabetes and tuberculosis and the implications for patient services. Lancet Diabetes Endocrinol. 2014;2(9):740-753.

15. Rock KL, Kataoka H, Lai JJ. Uric acid as a danger signal in gout and its comorbidities. Nat Rev Rheumatol. 2013;9(1):13-23.

16. Alvarez-Lario B, Macarron-Vicente J. Is there anything good in uric acid? QJM. 2011;104(12):1015-1024.

17. Bardin T, Richette P. Definition of hyperuricemia and gouty conditions. Curr Opin Rheumatol. 2014;26(2):186-191.

18. Ghaemi-Oskouie F, Shi Y. The role of uric acid as an endogenous danger signal in immunity and inflammation. Curr Rheumatol Rep. 2011; 13(2):160-166.

19. Kim SY, Guevara JP, Kim KM, Choi HK, Heitjan DF, Albert DA. Hyperuricemia and coronary heart disease: a systematic review and meta-analysis. Arthritis Care Res (Hoboken). 2010;62(2):170-180.

20. Kim SY, Guevara JP, Kim KM, Choi HK, Heitjan DF, Albert DA. Hyperuricemia and risk of stroke: a systematic review and meta-analysis. Arthritis Rheum. 2009;61(7):885-892.

International Journal of COPD

\section{Publish your work in this journal}

The International Journal of COPD is an international, peer-reviewed journal of therapeutics and pharmacology focusing on concise rapid reporting of clinical studies and reviews in COPD. Special focus is given to the pathophysiological processes underlying the disease, intervention programs, patient focused education, and self management protocols.
21. Horsfall LJ, Nazareth I, Petersen I. Serum uric acid and the risk of respiratory disease: a population-based cohort study. Thorax. 2014;69(11): 1021-1026.

22. Braghiroli A, Sacco C, Erbetta M, Ruga V, Donner CF. Overnight urinary uric acid: creatinine ratio for detection of sleep hypoxemia. Validation study in chronic obstructive pulmonary disease and obstructive sleep apnea before and after treatment with nasal continuous positive airway pressure. Am Rev Respir Dis. 1993;148(1):173-178.

23. Garcia-Pachon E, Padilla-Navas I, Shum C. Serum uric acid to creatinine ratio in patients with chronic obstructive pulmonary disease. Lung. 2007; 185(1):21-24.

24. Fabbri LM, Hurd SS. Global strategy for the diagnosis, management and prevention of COPD: 2003 update. Eur Respir J. 2003;22(1):1-2.

25. (GOLD) GIfCOLD. Global Strategy for the Diagnosis, Management, and Prevention of Chronic Obstructive Pulmonary Disease 2013. Global Initiative for Chronic Obstructive Lung Disease (GOLD); 2013. Available from: www.goldcopd.com

26. Suissa S, Dell'Aniello S, Ernst P. Long-term natural history of chronic obstructive pulmonary disease: severe exacerbations and mortality. Thorax. 2012;67(11):957-963.

27. Ställberg B, Janson C, Johansson G, et al. Management, morbidity and mortality of COPD during an 11-year period: an observational retrospective epidemiological register study in Sweden (PATHOS). Prim Care Respir J. 2014;23(1):38-45.

28. Williams MC, Murchison JT, Edwards LD, et al; Evaluation of COPD Longitudinally to Identify Predictive Surrogate Endpoints (ECLIPSE) Investigators. Coronary artery calcification is increased in patients with COPD and associated with increased morbidity and mortality. Thorax. 2014;69(8):718-723.

29. Bolland MJ, Grey A, Gamble GD, Reid IR. The effect of vitamin D supplementation on skeletal, vascular, or cancer outcomes: a trial sequential meta-analysis. Lancet Diabetes Endocrinol. 2014;2(4):307-320.

30. Odone A, Houben RM, White RG, Lonnroth K. The effect of diabetes and undernutrition trends on reaching 2,035 global tuberculosis targets. Lancet Diabetes Endocrinol. 2014;2(9):754-764.

31. Nicks ME, O'Brien MM, Bowler RP. Plasma antioxidants are associated with impaired lung function and COPD exacerbations in smokers. COPD. 2011;8(4):264-269.

32. Domej W, Oettl K, Renner W. Oxidative stress and free radicals in COPD - implications and relevance for treatment. Int J Chron Obstruct Pulmon Dis. 2014;9:91207-91224.

33. Tse HN, Tseng CZ. Update on the pathological processes, molecular biology, and clinical utility of $\mathrm{N}$-acetylcysteine in chronic obstructive pulmonary disease. Int J Chron Obstruct Pulmon Dis. 2014;9:825-836.

34. Billiet L, Doaty S, Katz JD, Velasquez MT. Review of hyperuricemia as new marker for metabolic syndrome. ISRN Rheumatol. 2014;2014:852954.

35. Bartziokas K, Papaioannou AI, Loukides S, et al. Serum uric acid as a predictor of mortality and future exacerbations of COPD. Eur Respir J. 2014;43(1):43-53.

36. Sato N, Kurashima K, Ubukata M, et al. [Prognostic significance of serum uric acid in patients with chronic obstructive pulmonary disease receiving home oxygen therapy]. Nihon Kokyuki Gakkai Zasshi. 2003; 41(2):74-80.

37. Plywaczewski R, Bednarek M, Jonczak L, Gorecka D, Sliwiniski P. Hiperurykemia u kobiet z obturacyjny bezdech senny [Hyperuricaemia in males with obstructive sleep apnoea (osa)]. Pneumonol Alergol Pol. 2005;73(3):254-259. Polish.

\section{Dovepress}

This journal is indexed on PubMed Central, MedLine and CAS. The manuscript management system is completely online and includes a very quick and fair peer-review system, which is all easy to use. Visit http://www.dovepress.com/testimonials.php to read real quotes from published authors. 